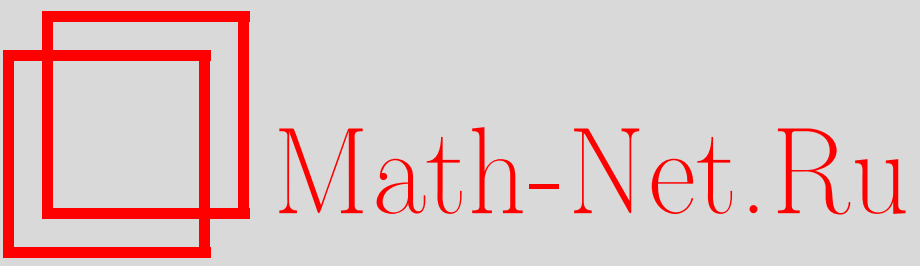

А. В. Егоров, K теории характеристических функций, УМН, 2004, том 59, выпуск $3,167-168$

DOI: https://doi.org/10.4213/rm747

Использование Общероссийского математического портала Math-Net.Ru подразумевает, что вы прочитали и согласны с пользовательским соглашением

http://www.mathnet.ru/rus/agreement

Параметры загрузки:

IP : 44.207 .124 .84

26 апреля 2023 г., 08:10:57 


\title{
К ТЕОРИИ ХАРАКТЕРИСТИЧЕСКИХ ФУНКЦИЙ
}

\author{
А. В. Егоров
}

Плотностью распределения вероятностей будем назьвать произвольную функцию вещественного переменного $p(x)$, удовлетворяющую условиям неотрицательности $p(x) \geqslant 0, x \in \mathbb{R}$, и нормировки $\int_{-\infty}^{+\infty} p(x) d x=1$. Характеристической функцией плотности $p(x)$ называется функция $f(t)$, определенная равенством $f(t)=\int_{-\infty}^{+\infty} e^{i t x} p(x) d x$. Из условия нормировки следует равенство $f(0)=1$.

К С.Н. Бернштейну восходит теорема о представлении вполне монотонных функций. Наиболее простое доказательство предложено в книге [1, с. 495].

ТЕОРема (С.Н. Бернштейн). Функиия $p(\lambda)$ на полуоси $(0, \infty)$ является преобразованием Лапласа некоторого распределения вероятностей тогда и только тогда, когда она вполне монотонна, т.е. имеет производные всех порядков $u(-1)^{n} p^{(n)}(\lambda) \geqslant 0, \lambda>0$, $u p(0)=1$.

Из этой теоремы вьведем следующее утверждение.

ТеОРема. На вещественной оси рассмотрим бесконечно дифференцируемую и абсолютно интегрируемую четную функцию $\varphi(t)$, которая принимает вещественнье значения. Пусть функиия $\varphi(t)$ удовлетворяет условию $\varphi^{(m)}(t) t^{-2}=O(1), t \rightarrow \infty$, и преобразование Фурье $\widehat{\varphi}$ принадлежит пространству $L^{1}(\mathbb{R})$. Функция $\varphi(t)$ является характеристической тогда и только тогда, когда

1) для всех $0<\delta<\infty$ и всех $p=0,1,2, \ldots$ справедливы неравенства

$$
\begin{aligned}
& \Theta_{p}(\delta)=(-1)^{p} \int_{0}^{\infty} \frac{1}{\delta+t^{2}} \varphi^{(2 p)}(t) d t \geqslant 0, \\
& \Psi_{p}(\delta)=(-1)^{p+1} \int_{0}^{\infty} \frac{1}{\delta+t^{2}} \varphi^{(2 p+1)}(t) t d t \geqslant 0 ;
\end{aligned}
$$

2) удовлетворяется равенство $\varphi(0)=1$.

ДокАЗАТЕЛЬСтво. В условиях нашей теоремы функция $\varphi(t)$ является характеристической тогда и только тогда, когда $\varphi(0)=1$ и функция $\widehat{\varphi}(\omega)=\frac{1}{2 \pi} \int_{-\infty}^{+\infty} e^{-i t \omega} \varphi(t) d t$ неотрицательна для всех $\omega \in \mathbb{R}$. Действительно, в силу теоремы обращения для преобразований Фурье функция $\widehat{\varphi}(\omega)$ задает плотность распределения вероятностей, соответствующего характеристической функции $\varphi(t)$. Здесь использована абсолютная интегрируемость $\varphi(t)$. В силу четности, $\varphi(t)=$ $\varphi(-t)$, эту формулу можно записать так $\widehat{\varphi}(\omega)=\frac{1}{\pi} \int_{0}^{\infty} \varphi(t) \cos (\omega t) d t$. Согласно теореме С.Н. Бернштейна функция $\widehat{\varphi}(\omega)$ тогда и только тогда неотрицательна на полуоси $[0, \infty)$, когда ее преобразование Лапласа $\pi \cdot \mathbf{L} \widehat{\varphi}(\omega)=\Phi(\lambda)=\pi \int_{0}^{\infty} \widehat{\varphi}(\omega) \exp (-\lambda \omega) d \omega$ оказьвается вполне монотонной функцией. Напомним, что гладкая функция $\Phi(\lambda)$ называется вполне монотонной, если при $0<\lambda<\infty$ все ее четные производные $\Phi^{(2 p)}(\lambda)$ неотрицательны, а все нечетные $\Phi^{(2 p+1)}(\lambda)$ неположителшны.

Имеют место равенства

$$
\begin{aligned}
\pi \cdot \mathbf{L} \widehat{\varphi}(\omega)=\Phi(\lambda) & =\int_{0}^{\infty} \int_{0}^{\infty} \varphi(t) \cos (\omega t) d t \exp (-\lambda \omega) d \omega \\
& =\int_{0}^{\infty} \varphi(t) \int_{0}^{\infty} \cos (\omega t) \exp (-\lambda \omega) d \omega d t \\
\int_{0}^{\infty} \cos (\omega t) \exp (-\lambda \omega) d \omega & =-\left.\frac{\lambda \cos (t \omega)-t \sin (t \omega)}{\exp (\lambda \omega)\left(\lambda^{2}+t^{2}\right)}\right|_{0} ^{\infty}=\frac{\lambda}{\lambda^{2}+t^{2}} \\
\Phi(\lambda) & =\int_{0}^{\infty} \frac{\lambda}{\lambda^{2}+t^{2}} \varphi(t) d t
\end{aligned}
$$


Изменение порядка интегрирования обосновано, поскольку при фиксированном значении $\lambda$ внутренние интегралы сходятся равномерно по параметрам в любом конечном промежутке, а повторные интегралы сходятся абсолютно в силу абсолютной интегрируемости функции $\varphi(t)$. Поэтому вьполнены условия теоремы о перестановке двух несобственных интегралов [2; с. 715].

Интегрируя по частям при фиксированном $\lambda>0$, получаем

$$
\begin{aligned}
\Phi(\lambda) & =\int_{0}^{\infty} \frac{\lambda}{\lambda^{2}+t^{2}} \varphi(t) d t=-\int_{0}^{\infty} \varphi(t) d \operatorname{arctg}_{*}\left(\frac{\lambda}{t}\right) \\
& =-\left.\varphi(t) \operatorname{arctg}_{*}\left(\frac{\lambda}{t}\right)\right|_{0} ^{\infty}+\int_{0}^{\infty} \operatorname{arctg}_{*}\left(\frac{\lambda}{t}\right) \varphi^{\prime}(t) d t=\frac{\varphi(0) \pi}{2}+\int_{0}^{\infty} \operatorname{arctg}_{*}\left(\frac{\lambda}{t}\right) \varphi^{\prime}(t) d t .
\end{aligned}
$$

3 десь $\operatorname{arctg}_{*}(\lambda / t)-$ гладкая функция на $[0, \infty)$, равная $\operatorname{arctg}(\lambda / t)$ при $t>0$ и $\pi / 2$ при $t=0$.

Дифференцируя интеграл по параметру $\lambda$, получим выражение для первой и второй производной. Эта операция законна, так как подьнтегральная функция и ее частная производная по $\lambda$ непрерывны по совокупности переменных $(\lambda>0)$ и продифференцированный интеграл равномерно сходится по признаку Вейерштрасса ввиду абсолютной интегрируемости $\varphi(t)$,

$$
\Phi^{\prime}(\lambda)=\int_{0}^{\infty} \frac{t}{\lambda^{2}+t^{2}} \varphi^{\prime}(t) d t=\int_{0}^{\infty} \frac{1}{\lambda^{2}+t^{2}} \varphi^{\prime}(t) t d t, \quad \Phi^{\prime \prime}(\lambda)=-\int_{0}^{\infty} \frac{2 \lambda t}{\left(\lambda^{2}+t^{2}\right)^{2}} \varphi^{\prime}(t) d t .
$$

Последний интеграл выгислим интегрированием по частям

$$
\begin{aligned}
\Phi^{\prime \prime}(\lambda) & =\int_{0}^{\infty} \varphi^{\prime}(t) d \frac{\lambda}{\lambda^{2}+t^{2}}=\left.\varphi^{\prime}(t) \frac{\lambda}{\lambda^{2}+t^{2}}\right|_{0} ^{\infty}-\int_{0}^{\infty} \frac{\lambda}{\lambda^{2}+t^{2}} \varphi^{\prime \prime}(t) d t \\
& =-\int_{0}^{\infty} \frac{\lambda}{\lambda^{2}+t^{2}} \varphi^{\prime \prime}(t) d t .
\end{aligned}
$$

Заметим, что здесь использовано равенство $\varphi^{\prime}(0)=0$.

Мы видим периодичность, состоящую в инвариантности формы функции $\Phi(\lambda)$ при повторном дифференцировании. Ее вторая производная задается интегралом такого же вида, но с обратньм знаком и подынтегралшная функция $\varphi(t)$ заменяется на $\varphi^{\prime \prime}(t)$.

Функция $\varphi^{\prime \prime}(t)$ четна, бесконечно дифференцируема, и все рассуждения могут быть повторены. Член вида $\left.\varphi^{(2 p+1)}(t) \frac{\lambda}{\lambda^{2}+t^{2}}\right|_{0} ^{\infty}$ исчезает в силу наложенных на $\varphi(t)$ условий четности и роста на бесконечности. Рекурсивно вычисляем последовательность производных

$$
\begin{array}{llrl}
\Phi^{(0)}(\lambda) & =\int_{0}^{\infty} \frac{\lambda}{\lambda^{2}+t^{2}} \varphi^{(0)}(t) d t, & & \Phi^{(1)}(\lambda)=\int_{0}^{\infty} \frac{1}{\lambda^{2}+t^{2}} \varphi^{(1)}(t) t d t, \\
\Phi^{(2)}(\lambda) & =-\int_{0}^{\infty} \frac{\lambda}{\lambda^{2}+t^{2}} \varphi^{(2)}(t) d t, & \Phi^{(3)}(\lambda)=-\int_{0}^{\infty} \frac{1}{\lambda^{2}+t^{2}} \varphi^{(3)}(t) t d t \text { и т.д. }
\end{array}
$$

Таким образом, при $\lambda>0$ знак четной производной $\Phi^{(2 p)}(\lambda)$ совпадает со знаком функции $\Theta_{p}(\lambda)=(-1)^{p} \int_{0}^{\infty} \frac{1}{\lambda^{2}+t^{2}} \varphi^{(2 p)}(t) d t$, а знак нечетной производной $\Phi^{(2 p+1)}(\lambda)$ противоположен знаку функции $\Psi_{p}(\lambda)=(-1)^{p+1} \int_{0}^{\infty} \frac{1}{\lambda^{2}+t^{2}} \varphi^{(2 p+1)}(t) t d t$. Полная монотонность преобразования Лапласа, равносильная неотрицательности плотности $\widehat{\varphi}(\omega)$ и характеристичности $\varphi(t)$, эквивалентна системе неравенств $\Theta_{p}(\delta) \geqslant 0, \Psi_{p}(\delta) \geqslant 0, p=0,1,2, \ldots$, где $\delta=\lambda^{2}$. Теорема доказана.

Автор выражает глубокую благодарность А. Н. Ширяеву и А. С. Черному за обсуждение результата данной работы.

\section{СПИСОК ЛИТЕРАТУРЫ}

[1] В. Феллер. Введение в теорию вероятностей и ее приложения. Т. 2. М.: Мир, 1984. [2] Г. М. Фихтенгольц. Курс дифференциального и интегрального исчисления. Т. ІІ. М.: Наука, 1969. 